\title{
Realistc Detailed Balance Study of the Quantum Efficiency of Quantum Dot Solar Cells
}

\author{
Alexander Mellor, Antonio Luque, Ignacio Tobías, and Antonio Martí
}

\begin{abstract}
An attractive but challenging technology for high efficiency solar energy conversion is the intermediate band solar cell (IBSC), whose theoretical efficiency limit is $63 \%$, yet which has so far failed to yield high efficiencies in practice. The most advanced IBSC technology is that based on quantum dots (QDs): the QD-IBSC. In this paper, k.p calculations of photon absorption in the QDs are combined with a multi-level detailed balance model. The model has been used to reproduce the measured quantum efficiency of a real QD-IBSC and its temperature dependence. This allows the analysis of individual sub-bandgap transition currents, which has as yet not been possible experimentally, yielding a deeper understanding of the failure of current QD-IBSCs. Based on the agreement with experimental data, the model is believed to be realistic enough to evaluate future QD-IBSC proposals.
\end{abstract}

\section{Introduction}

Over 50 years ago, Shockley and Queisser (SQ) demonstrated that conventional single-bandgap solar cells were ultimately limited to $41 \%$ efficiency. ${ }^{[1]}$ Since then, many technologies have been proposed (and developed with different degrees of success) with the aim of superseding this limit. ${ }^{[2]}$ One attractive but challenging concept is the intermediate band solar cell (IBSC), whose detailed balance efficiency limit has been calculated as $63 \% .^{[3]}$

The concept is based on the introduction of an intermediate band (IB) between the valence band (VB) and conduction band (CB) of a semiconductor. The IB allows an increase in photocurrent via a sequential two sub-bandgap photon absorption process in which one photon promotes an electron from the VB to the IB and another promotes an electron from the IB to the $\mathrm{CB}$, creating a single electron-hole pair.

IBSCs have been realized in which the IB is constituted by the bound-state energy levels of InAs quantum dots (QDs) in a GaAs matrix. ${ }^{[4-6]}$ This system has non-optimal bandgaps, and usually presents several IB levels within the GaAs forbidden band, which is not ideal. ${ }^{[7]}$ Nonetheless, it has allowed the basic principles of IB operation to be proven. ${ }^{[8]}$

Two main failings of present QD-IBSCs have been identified to prevent them from reaching high efficiencies. Firstly, the sub-bandgap photocurrent generated due to the presence of the QDs is very small. Secondly, the presence of the QDs leads to a reduction in the open circuit voltage $\left(V_{\text {oc }}\right)$. Consequently, the efficiency of fabricated QD-IBSCs tends to be less than that of an equivalent reference cell with no QDs. ${ }^{[4,5]}$

In order to better understand the problems facing existing QD-IBSCs, there have been several recent theoretical studies into the absorption of subbandgap photons by the QDs. Optical matrix elements have been calculated for intraband $(\mathrm{IB} \leftrightarrow \mathrm{CB})^{[9,10]}$ and interband $(\mathrm{VB} \leftrightarrow \mathrm{IB})^{[11,12]}$ transitions using, in the former case, a one-band $\mathrm{k} \cdot \mathrm{p}$ method, and, in the latter case, the so-called empirical $\mathrm{k} \cdot \mathrm{p}$ Hamiltonian (EKPH) method, ${ }^{[11]}$ which is a four-band $\mathrm{k} \cdot \mathrm{p}$ method.

Sub-bandgap photon absorption/emission depends not only on the optical matrix elements, but on the electron occupation of the different bands and levels in the system. Neglecting nonradiative processes, these can be calculated by considering the so-called detailed balance of absorption and emission for each electronic transition and exacting the continuity of generation and recombination currents via each IB level. Detailed balance considerations have been used in the past to calculate the aforementioned efficiency limits of IBSCs $^{[3]}$ and single bandgap solar cells, ${ }^{[1]}$ but in these cases, full absorption of photons in each transition was assumed. In this work, we do not assume full absorption, but instead take the optical matrix elements calculated in the previous works as input for the detailed balance model. This allows a realistic estimate of the sub-bandgap current generated by the QD-IBSC in the radiative limit.

In this work, the model has been applied to calculation of the internal quantum efficiency (IQE) of a real QD-IBSC and its temperature dependence. The results are compared to previously published experimental data, ${ }^{[13,14]}$ with good agreement. Historically, this experiment has served two main purposes. It confirmed that the sub-bandgap photocurrent is too low, and established the thermal escape of electrons from the IB to the $\mathrm{CB}$ as a cause of the reduction of $V_{\mathrm{oc}}$ with respect to an equivalent reference cell with no QDs.

Using the model, we can analyse the individual sub-bandgap transition currents that make up the measured IQE, something that is not possible experimentally. We confirm that the poor sub-bandgap photocurrent is a consequence of the weak photon absorption investigated in the previous works. ${ }^{\left[{ }^{[-12]}\right.}$ We also confirm that the thermal escape can be explained by radiative processes involving thermal photons at $300 \mathrm{~K}$. This effect has 
been previously investigated considering the electronic system submerged in a reservoir of thermal photons at room temperature. ${ }^{[15]}$ In this study, the thermal photons are incident on the active IB layer from the surroundings and from the GaAs substrate.

Finally, we believe that the good agreement with experimental results makes this model a useful tool for evaluating future proposals for QD-IBSC prototypes, particularly (but not only) in terms of the materials used and the QD dimensions. Therefore, a complete derivation is given in the Supporting Information of this article. The model is valid for QD-IBSCs operating in the radiative limit, as is the case at short circuit. Close to the operating voltage, non-radiative processes such as Shockley-Read-Hall (SRH) recombination become important. ${ }^{[16]}$ However, SRH can be minimized by improving material quality. Results of the model at operating voltage therefore correspond to the best that be achieved by a proposed QD-IBSC.

The paper is organized as follows. In Section 2, a summary of the detailed balance model is given, along with the input parameters including the calculated absorption coefficients. In Section 3, modelling results are presented of the internal quantum efficiency (IQE) at different temperatures and comparison is made to previously published experimental results. The different intermediate transitions are analysed in detail. In Section 4, conclusions are drawn. A complete derivation of the detailed balance model is given in the Supporting Information.

\section{The Detailed Balance Model}

Here, a summary of the detailed balance model is given, which should be sufficient for the understanding of the results. A reader wishing to read the full derivation in the Supporting Information should read this summary first.

The QD-IBSC simulated in this work is the sample denoted $\mathrm{SB}^{[13]}$ and S3. ${ }^{[14]}$ This system consisted of a 30-layer stack of $\operatorname{In}(\mathrm{Ga}) \mathrm{As} / \mathrm{GaAs}$ QDs, with a surface density of $4 \times 10^{10} \mathrm{~cm}^{-2}$, grown in the Stranski-Krastanov mode on a GaAs substrate. The QDs had the shape of truncated quadrangular pyramids with dimensions of $16 \mathrm{~nm} \times 16 \mathrm{~nm} \times 6 \mathrm{~nm}$. These have been modelled as square based parallelepipeds when calculating the confined state energy levels and optical matrix elements. ${ }^{\left[{ }^{-12}\right]}$ Further details of the growth conditions of the real samples can be found elsewhere. ${ }^{[4,13]}$ This type of QD-IBSC is the most extensively investigated to date; we therefore refer to the investigated sample as the state-of-the-art (SOTA) cell throughout this paper.

\subsection{Balance Equations}

The SOTA cell consists of an InAs/GaAs QD stack (the IB layer) sandwiched between two conventional GaAs layers. A simplified band diagram of a single QD in the SOTA cell is shown in Figure 1. The grey lines represent the VB and CB band edges, the offsets being due to the InAs QD.

The confining potential in the CB supports three discrete bound state levels in the energy range of the host forbidden band. Following the nomenclature found in the literature, ${ }^{[9]}$ these are labelled IB(111), IB(211) and IB(221). The IB(211) level
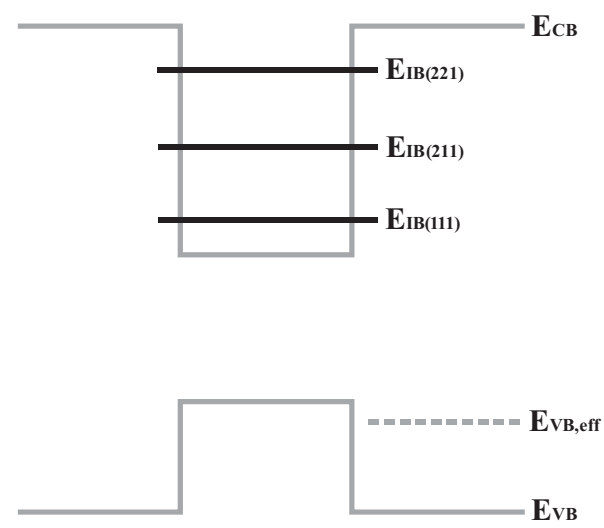

Figure 1. A simplified band diagram of a single QD in the SOTA cell. Upper grey line: conduction band edge. Lower grey line: valence band edge. Black lines: confined state energy levels whose energy is within the host forbidden band. Dashed grey line: effective valence band edge.

corresponds to the pair of states IB(211)/IB(121), which are degenerate due to the QDs' reflection symmetry in the $x-y$ plane. The energy of each level is denoted $E_{\mathrm{IB}(111)}, E_{\mathrm{IB}(211)}$, and $E_{\mathrm{IB}(221)}$. It is not a priori assumed that the IB levels are thermally coupled and as such they are described by Fermi-Dirac functions with three distinct quasi Femi levels (QFLs): $E_{\mathrm{F}, \mathrm{IB}(111)}, E_{\mathrm{F}, \mathrm{IB}(211)}$ and $E_{\mathrm{F}, \mathrm{IB}(221)}$.

The onset of the host $\mathrm{CB}$ is denoted $E_{\mathrm{CB}}$. Above this energy, there is a continuum of extended states and a set of discrete virtual bound states ${ }^{[17]}$ (neither pictured), all of which are described by a single QFL denoted $E_{\mathrm{F}, \mathrm{CB}}$.

The confining potential pedestal in the VB supports numerous bound heavy-hole and light-hole states, (not pictured). The heavy-hole states are so close together as to form a pseudo continuum, and it is therefore assumed that all confined hole states are thermally coupled to one another and to the rest of the VB. The VB is therefore considered to have its energy onset at the confined heavy-hole ground state energy, which is near the top of the pedestal ( $E_{\mathrm{VB}, \text { eff }}$, dashed line). The Fermi-Dirac distribution of the VB is described by a single QFL, denoted $E_{\mathrm{F}, \mathrm{VB}}$. The lower density of states in the VB pedestal is taken into account.

We therefore have five distinct electron populations: that of the $\mathrm{CB}$, that of the VB (whose upper energy onset is at $E_{\mathrm{VB}, \mathrm{eff}}$ ), and those of the three discrete IB energy levels. Generation and recombination currents exist between the five populations due to the respective emission and absorption of photons. The net generation current (generation or upward traffic of electrons up minus recombination or downward traffic) between a given pair of bands/levels is denoted $J_{l \rightarrow u}$, where $l$ and $u$ are, respectively, the lower and upper level or band. Due to the conventional GaAs layers on either side of the QD stack, current can only be extracted via the VB and CB and not directly from the IB levels. Hence, for each IB level with index $k$, we can write a current balance equation:

$\sum_{l} J_{l \rightarrow k}-\sum_{u} J_{k \rightarrow u}=0$

where the first and second sums are made over all levels (or bands) whose energy is, respectively, lower or higher than that 
of IB level $k$. Equation 1 presents three equations, one for each intermediate band level.

Charge neutrality is assumed to in the QD stack. This condition is found in the inside (far from the edges) of thick stacks of QDs, as is the case for the QD-IBSC under investigation. ${ }^{18]}$ We can therefore write

$n_{C B}-p_{V B}+n_{I B}-N_{d}^{+}=0$

where $n_{\mathrm{CB}}$ and $p_{\mathrm{VB}}$ are the concentrations of electrons and holes in the $\mathrm{CB}$ and $\mathrm{VB}$, respectively (taking also the confined hole states into account), $n_{\mathrm{IB}}$ is the electron concentration in the several IB states (summed), and $N_{d}^{+}$is the concentration of ionized donor atoms used to prefill the IB. The QD layer of the SOTA cell is $\delta$-doped to a concentration that supplies one donor atom per QD. ${ }^{[13]}$ The number of donor atoms is therefore equal to the number of QDs. The number of ionized donor atoms is given in Equation S26 in the Supporting Information, along with expressions for all terms in Equation 2.

Finally, we make the simplifying assumption that the terminal voltage is equal to the split between the VB and CB QFLs. This is equivalent to assuming infinite carrier mobility and, therefore, zero series resistance.

$E_{F, C B}-E_{F, V B}=q_{e} V$

All the terms in Equations 1-3 (given explicitly in the Supporting Information) are dependent on the five unknown QFLs of the system, along with a number of input parameters described in the following sub-section. We have five equations in five unknowns that can be solved numerically to yield the QFLs. Once the QFLs are known, all net generation currents can be calculated, yielding the overall sub-bandgap current of the device.

For a given pair of bands/levels, $J_{l \rightarrow u}$ is given by the expression (derivation in Supporting Information-please observe that the bracketed expression in the second line forms part of the integrand):

$$
\begin{aligned}
& J_{l \rightarrow u}=q_{e} W \int_{0}^{E_{g}} d E_{p h o t}\left(f_{l}-f_{u}\right) \\
& \left(\Phi_{i n c} \alpha_{x, Y}^{\max }+\frac{8 \pi n^{2} E_{p h o t}^{2}}{3 h^{3} c^{2}}\left(f_{B, 0 T_{c}}-f_{B, \mu T_{c}}\right)\left(2 \alpha_{x, y}^{\max }+\alpha_{z}^{\max }\right)\right)
\end{aligned}
$$

where $f_{1}$ and $f_{\mathrm{u}}$ are respectively the Fermi-Dirac function describing the electron occupancies of the lower and upper level. $\alpha_{x, y}^{\max }$ and $\alpha_{z}^{\max }$ are the absorption coefficient of the specific transition for photons polarized in the $x y$ plane and in the $z$ direction respectively. These are related to the optical matrix elements for the given transition as described in Equations S16 in the Supporting Information (due to the $x y$ symmetry of the QDs we have $\left.\alpha_{x, y}^{\max }:=\alpha_{x}^{\max }=\alpha_{y}^{\max }\right)$. The superscript " $m a x$ " indicates that these are the values before modification by the electron occupancies of the lower and upper levels. They are related to the measurable absorption coefficient by

$\alpha^{\max }=\alpha /\left(f_{l}-f_{u}\right)$

$\Phi_{i n c}$ is the incident photon flux. The first term in the long bracketed expression in Equation 4 therefore represents the absorption of external illumination. $f_{B, 0 T_{c}}$ and $f_{B, \mu T_{c}}$ are Bose-Einstein distributions at chemical potentials of zero and $\mu=E_{F, u}-E_{F, l}$ respectively (both at the cell/ambient temperature $T_{\mathrm{c}}$ ). The term proportional to $f_{B, 0 T_{c}}$ represents the absorption of thermal photons incident on the IB layer from the surroundings. The term proportional to $f_{B, \mu} T_{\mathcal{C}}$ represents the emission of luminescent photons into the surroundings.

Luminescent photons necessarily escape the IB layer through the same paths that thermal photons enter. These paths are into/from the air at the front through a narrow escape cone, and into/from the thick GaAs substrate at the rear through the entire hemisphere (the substrate and IB layer have the same refractive index). It is reasonable to think that below-bandgap photons entering from the doped substrate have zero chemical potential under any voltage conditions since they mainly interact with electrons or holes at thermal equilibrium.

\subsection{Input Parameters}

For the SOTA cell, the optical matrix elements have been calculated in other works ${ }^{[9-12]}$ The resulting $\alpha_{x, y}^{\max }$ and $\alpha_{z}^{\max }$ used as the input for the calculations are plotted in Figure 2. The Diracdelta in Equations S16 has been approximated as a Gaussian with a deviation of $25 \mathrm{meV}$ to account for the variance of QD sizes in the layer stack. The $\operatorname{IB}(111) \leftrightarrow \operatorname{IB}(221)$ transition is
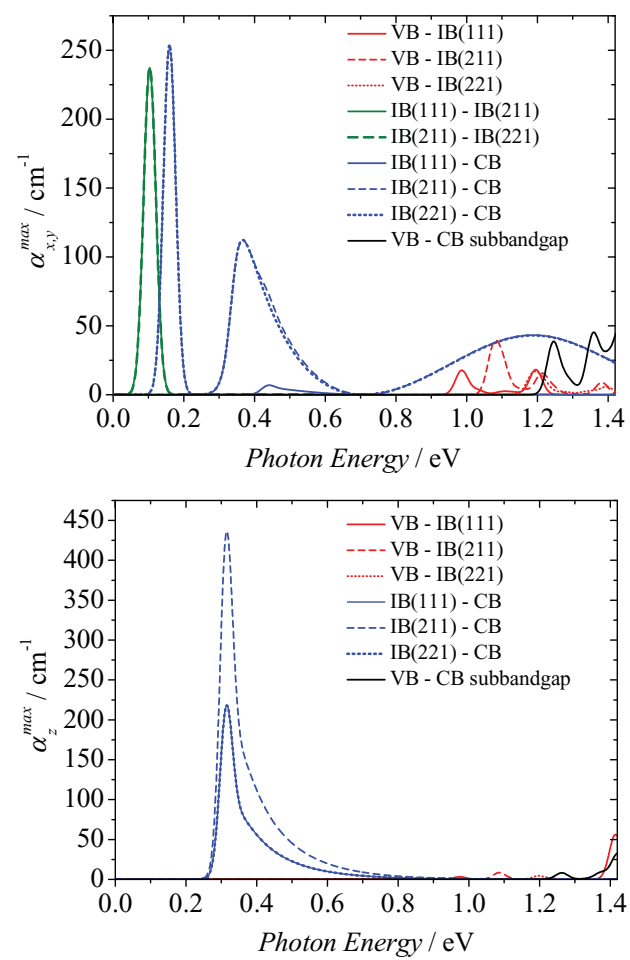

Figure 2. Absorption coefficients for the different transitions in the SOTA cell before modification by the electron occupancies of the lower and upper levels. Top: absorption coefficients for photons polarized in the xy plane. Bottom: absorption coefficient for photons polarized in the $z$ direction. Each curve represents a different electronic transition between all the bands and levels shown in Figure 1. Note that in the upper graph, the $I B(211)-C B$ and $I B(221)-C B$ absorption coefficients are practicallty identical, whereas in the lower graph, the $I B(111)-C B$ and $I B(211)-C B$ absorption coefficients are identical. 
Table 1. Input parameters used in detailed balance model.

\begin{tabular}{lcc}
\hline Symbol & Value & Units \\
\hline$E_{\mathrm{CB}}$ & 0 & $\mathrm{eV}$ \\
$E_{\mathrm{VB}}$ at $300 \mathrm{~K}$ & 1.42 & $\mathrm{eV}$ \\
$E_{\mathrm{VB}, \text { eff }}$ at $300 \mathrm{~K}$ & -1.24 & $\mathrm{eV}$ \\
$E_{\mathrm{IB}(111)}$ & -0.26 & $\mathrm{eV}$ \\
$E_{\mathrm{IB}(211)}$ & -0.16 & $\mathrm{eV}$ \\
$E_{\mathrm{IB}(221)}$ & -0.06 & $\mathrm{eV}$ \\
$W$ & 2.4 & $\mu \mathrm{m}$ \\
$v$ & 3.5 & \\
$\rho_{\mathrm{QD}}$ & $5 \times 10^{15}$ & $\mathrm{~cm}^{-3}$ \\
$N_{\mathrm{d}}$ & $5 \times 10^{15}$ & $\mathrm{~cm}^{-3}$ \\
$E_{\mathrm{d}}$ & -0.006 & $\mathrm{eV}$ \\
$N_{\mathrm{CB}}$ & $1.95 \times 10^{19} T_{\mathrm{c}}^{3 / 2}$ & $\mathrm{~cm}^{-3}$ \\
$N_{\mathrm{VB}}$ & $4.10 \times 10^{20} T_{\mathrm{c}}^{3 / 2}$ & $\mathrm{~cm}^{-3}$ \\
\hline
\end{tabular}

forbidden for $x, y$ polarization and all transitions between IB levels are forbidden for $\mathrm{z}$ polarization; ${ }^{[9]}$ hence, these are not plotted. "VB-CB sub-bangap" refers to transitions between the pseudo continuum of states in the VB pedestal and the CB, the transition energies for these being lower than the host bandgap $E_{\mathrm{g}}$.

The remaining input parameters used in the detailed balance model are listed in Table 1.

\section{Results}

The model has been used to simulate the experiments presented in other studies, ${ }^{[13,14]}$ where the IQE of the SOTA cell was measured at different temperatures. Before presenting the modelled results, we comment briefly on the relevance of the experimental results. In the articles, it was demonstrated that electrons pumped from the VB to the IB by external illumination can readily escape to the CB by thermal processes. Strong IB $\leftrightarrow \mathrm{CB}$ thermal escape implies that positive sub-bandgap photocurrent can only be delivered at a voltage limited by the VB-IB sub-bandgap, ${ }^{[19]}$ which is smaller than the overall VB-CB bandgap. This ultimately limits the conversion efficiency of the device at room temperature. A goal of QD-IBSC research has since been to minimize the thermal escape, for example by using a larger bandgap hosts. ${ }^{[20,21]}$

In the experiment, the temperature of the QD-IBSC and its immediate surroundings are controlled using a cryostat and the QD-IBSC was illuminated by a monochromator with a linewidth of around $1 \mathrm{~nm}$ and a total irradiance of $0.5 \mathrm{~mW} \mathrm{~cm}^{-2}$. In the model, the temperature is controlled by the parameter $T_{\mathrm{c}}$ and the incident flux is modelled as a narrow Gaussian function with a deviation of $\sigma=0.01 \mathrm{eV}$.

$$
\Phi_{i n c}\left(E_{\text {phot }}\right)=\frac{\exp \left(-\left(E_{\text {phot }}-E_{\text {mon }}\right)^{2} / \sigma\right)}{\sigma \sqrt{\pi}} \times 0.5 \mathrm{mWcm}^{-2}
$$

where $E_{\text {mon }}$ is the nominal output photon energy of the monochromator.
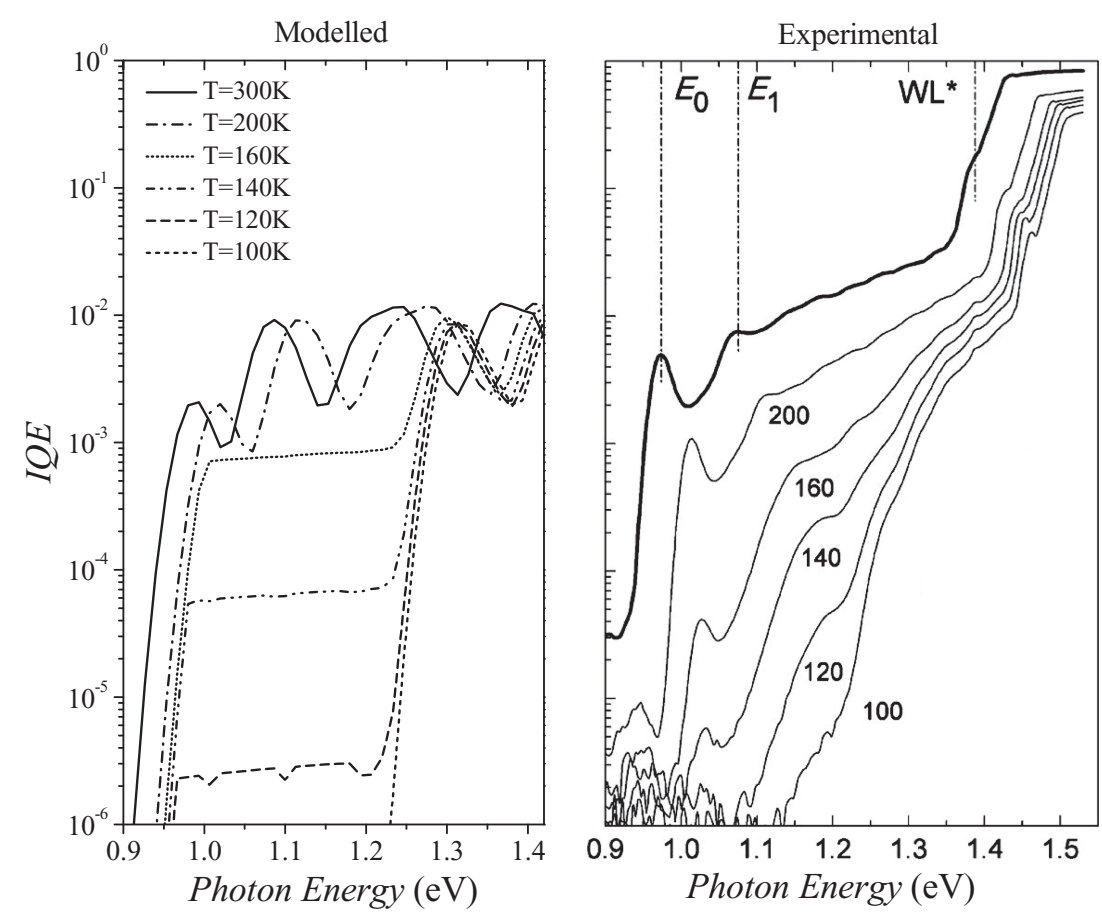

Figure 3. Temperature dependent IQE for the SOTA cell. Left: IQE calculated using the detailed balance model. Right: Measured IQE from other work. ${ }^{[13,14]}$ The photon energy on the horizontal scale refers to the nominal output photon energy of the monochromator ( $E_{\text {mon }}$ in Equation 4). Right figure Reprinted with permission. ${ }^{[13]}$ Copyright 2010, American Institute of Physics.

\subsection{Temperature Dependent Internal Quantum Efficiency}

Figure 3 (left) shows the calculated IQE for a range of cell temperatures. Figure 3 (right) shows the measured temperature dependent IQE published elsewhere. ${ }^{[13,14]}$ The peaks marked in the experimental curve as $E_{0}$ and $E_{1}$ are transitions from the VB to the ground and first excited states in the QD CB well; these correspond to states $\operatorname{IB}(1,1,1)$ and $\operatorname{IB}(2,1,1) /$ $(1,2,1)$ in the model. The quantitative agreement between the experimental IQE and that calculated using the EKPH method for energies below $1.35 \mathrm{eV}$ and at room temperature has been discussed in the literature. ${ }^{[11]}$ The fact that the weak sub-bandgap IQE has been reproduced by the model, which assumes infinite carrier mobility, demonstrates that this is a problem of weak photon absorption in the QDs and not of carrier extraction from the QD stack. The detailed balance model also reproduces qualitatively the temperature dependence of the IQE in the $0.9-1.2 \mathrm{eV}$ range. The quantitative agreement is discussed in the following subsection.

The modelled IQE ceases to be temperature dependent above around $1.2 \mathrm{eV}$. This is 
the effective bandgap of the IB layer caused by the quasi continuum of confined-hole states that invade the bandgap (see Figure 1). This can be seen somewhat in the measured data at lower temperatures. Both modelled and experimental data show a blue shifting of features at lower temperatures, caused by the widening of the host bandgap.

There are also features not reproduced by the model. The step in the experimental data around $1.35 \mathrm{eV}$ (labelled WL) is due to absorption by the quantum wells formed by the socalled wetting layer during QD growth. Also, the measured IQE increases steadily with increasing photon energy, an effect that becomes more pronounced at lower temperatures. This could be due to non-instantaneous relaxation of holes from the host valence band to the confined hole states in the QDs. Neither of these effects has been considered in the model.

\subsection{Arrhenius Plot}

To provide quantitative analysis, an Arrhenius plot of the modelled IQE at the $E_{0}$ peak is shown in Figure 4 (left) (squares), along with the measured Arrhenius plot from another work ${ }^{[13]}$ in Figure 4 (right). Both the modelled and experimental data show distinct high and low temperature regimes.

In the high temperature regime, the photocurrent is limited by $\mathrm{VB} \rightarrow \mathrm{IB}$ transitions induced by the monochromator illumination. The modelled data is entirely temperature independent in this regime, whereas the experimental appears to approach a plateau gradually on increasing the temperature. This discrepancy is also visible in Figure 3 (comparing results at $300 \mathrm{~K}$ and $200 \mathrm{~K})$. We consider there is a basic agreement; small discrepancies are to be expected given the simplicity of the model.

In both plots, the low temperature regime is linearly temperature dependent. Here the limitation is due to thermal escape from the IB to the CB. The linearity implies an Arrhenius type temperature dependence of the form $\exp \left(-E_{A} / k_{B} T_{c}\right)$, where $E_{\mathrm{A}}$ is the so-called activation energy of the thermal escape. Applying a linear fit to the modelled data in this regime yields and activation energy of $235 \mathrm{meV}$, to be compared to the $224 \mathrm{eV}$ yielded from the experimental data. In the model, the thermal escape occurs due to absorption of the aforementioned thermal photons incident on the IB layer from the surroundings and from the substrate, as was postulated elsewhere. ${ }^{[15]}$ Due to the good agreement between the measured thermal escape activation energy and that predicted by the model, we believe the model can serve as a useful tool for evaluating future proposals for QD geometries and material systems for QD-IBSCs.

The calculation has been repeated with a monochromator irradiance of $5 \mathrm{~mW} \mathrm{~cm}{ }^{-2}$, ten times higher than the estimated irradiance in the experiment. The results are plotted as triangles in Figure 4 (left). The change in irradiance causes a lateral shift in the temperature dependent part of the curve, achieving much better agreement with the experimental data. Although it is possible that the experimental irradiance is actually much higher than estimated, this discrepancy is more likely due to the calculated $E_{0}$ absorption peak being below the experimental one (as is visible in Figure 3).

In previous papers, it has often been stated that the thermal escape is non-optical. However, here, the thermal escape has been well reproduced using a model that considers only radiative transitions. This suggests that the thermal escape is therefore mainly due to interactions with thermal photons incident from the substrate and the surroundings.

\subsection{Analysis of the Individual Transitions}

Using the model, we can investigate the individual subbandgap currents that lead to the overall IQE. We define the IQE for each sub-bandgap transition as being the net number of charge carriers making that transition per incident photon, where positive and negative IQEs corresponds to net generation and recombination respectively. The IQE for each individual transition is plotted in Figure $\mathbf{5}$ (left) and (right) for temperatures of $300 \mathrm{~K}$ and $140 \mathrm{~K}$ respectively, these belonging to the two different regimes. Each figure is divided into three panels: the top panel shows transitions from the VB to the IB levels, the middle panel transitions between IB levels, and the bottom
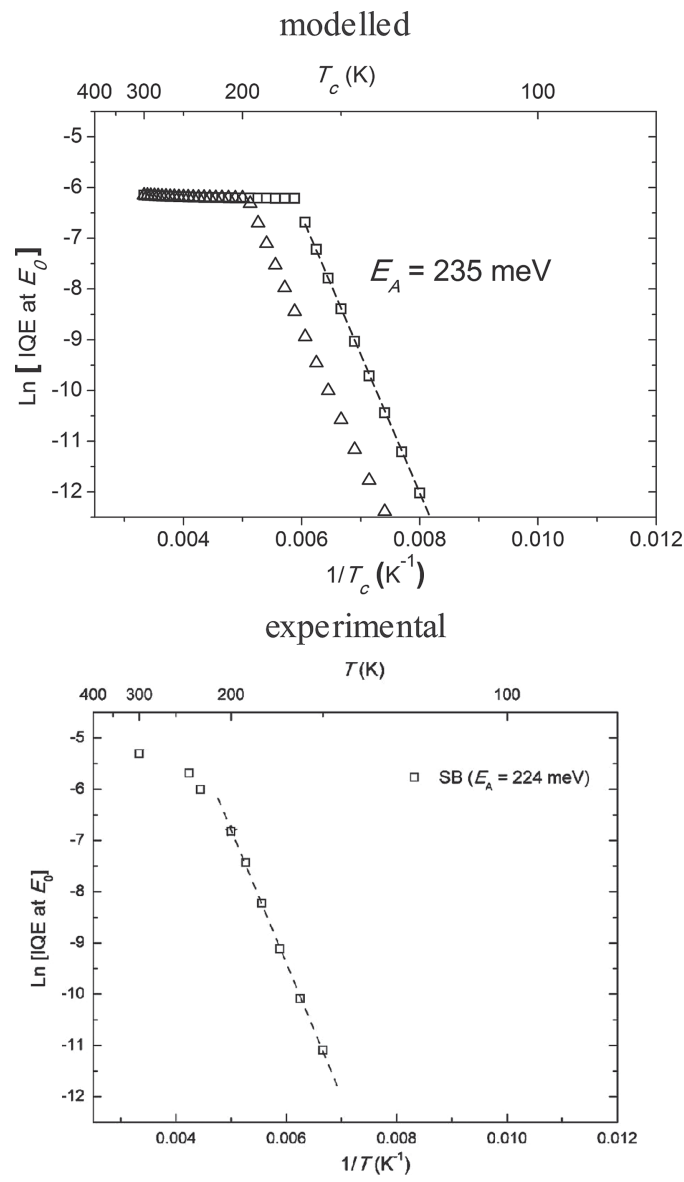

Figure 4. Arrhenius plots of the IQE at $E_{0}$ for the SOTA cell. Top graph: values calculated using detailed balance model. Squares: monochromator irradiance $=0.5 \mathrm{~mW} \mathrm{~cm}{ }^{-2}$. Triangles: monochromator irradiance $=5 \mathrm{~mW} \mathrm{~cm}{ }^{-2}$. Bottom: measured values published previously. ${ }^{[13]}$ In both graphs, the dashed lines are linear fits to the linear parts of the curves; the thermal activation energies EA are extracted from the slopes of these fits. Right figure reprinted with permission. ${ }^{[13]}$ Copyright 2010, American Institute of Physics. 

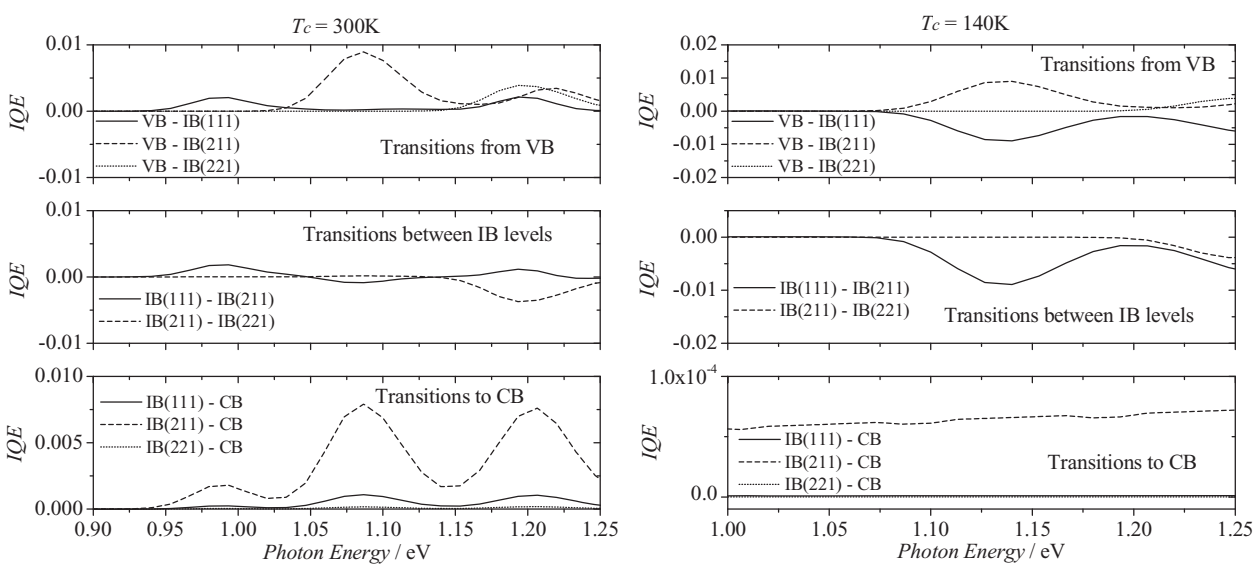

Figure 5. IQEs of the individual transitions in the multi-level system for the SOTA cell cell at different temperatures. Left: $T_{c}=300 \mathrm{~K}$. Right: $T_{\mathrm{c}}=$ $140 \mathrm{~K}$. The IQE here means the net number of charge carriers making the stated transition per incident photon. Negative values imply net recombination for the transition.

panel transitions from IB levels to the CB. Table 2 summarizes the dominant path by which electrons are delivered from the VB to the CB in each energy range, these are the same for both temperatures.

At $300 \mathrm{~K}$, the monochromator pumps electrons from the VB to one of the IB levels depending on the photon energy. The electrons in this level then make a transition to the IB(211) level due to absorption/emission of thermal photons. From the IB(211) level they are extracted to the CB, again due to absorption of thermal photons.

At $140 \mathrm{~K}$, the dominant paths by which electrons reach the $\mathrm{CB}$ via the IB states is the same as for $300 \mathrm{~K}$. However, due to the reduced radiative thermal escape at this temperature, the $\mathrm{IB}(211) \rightarrow \mathrm{CB}$ current is much weaker than at $300 \mathrm{~K}$ (note the different vertical scales). Consequently, most of the electrons reaching the IB levels from the VB recombine back to the VB via the IB(111) level.

These results reveal why it has as yet been impossible to measure optical transitions from the IB ground state to the CB experimentally. For normally incident photons $(x, y$ polarization), there is weak direct coupling from the IB(111) level to the $\mathrm{CB}$ (Figure 2 top). Hence, photogeneration via the IB(111) level is not a two photon process but a three photon process. Measuring this process at low temperatures would require photons of appropriate energy for each of the three transitions listed in the first row of Table 2 . What has probably been lacking in previous experiments is a source of low energy photons (around $100 \mathrm{meV}$ ) to pump from the $\mathrm{IB}(111)$ to the IB(211) level.

Table 2. Dominant paths by which electrons reach the $C B$ via the $I B$ states for differenent monochromator energy ranges. The paths are the same at $300 \mathrm{~K}$ and $160 \mathrm{~K}$.

\begin{tabular}{lc}
\hline $\begin{array}{c}\text { Energy Range } \\
{[\mathrm{eV}]}\end{array}$ & Dominant Path \\
\hline $0.95-1.02$ & $\mathrm{VB} \rightarrow \mathrm{IB}(111) \rightarrow \mathrm{B}(211) \rightarrow \mathrm{CB}$ \\
$1.02-1.15$ & $\mathrm{VB} \rightarrow \mathrm{IB}(211) \rightarrow \mathrm{CB}$ \\
$1.15-1.35$ & $\mathrm{VB} \rightarrow \mathrm{IB}(221) \rightarrow \mathrm{B}(211) \rightarrow \mathrm{CB}$ \\
\hline
\end{tabular}

Since radiative thermal escape always occurs via the IB(211) level and never directly from the ground IB(111) level, it is clear that the excited $\operatorname{IB}(211)$ and $\operatorname{IB}(221)$ states must be removed from the forbidden band to suppress the escape. One suggested means of doing this is to decrease the QD size. ${ }^{[22,23]}$ This increases the energy of all IB levels, pushing the IB(211) and $\operatorname{IB}(221)$ states into the host $C B$, where they become virtual bound states. ${ }^{[2]}$ However, the disadvantage is that the IB(111) energy also increases. This would place the $\mathrm{IB}(111) \leftrightarrow \mathrm{CB}$ transition at an energy at which thermal photons outnumber photons incident from the sun, and hence induce direct thermal escape from the IB(111) level. To effectively suppress thermal escape, it is therefore necessary to move to a material system with a larger $\mathrm{CB}$ offset, such as InAs/AlGaAs${ }^{[20]}$ or certain IV-VI/II-VI combinations, ${ }^{[21]}$ whilst simultaneously tuning the QD size so that all excited states are removed from the forbidden band.

\section{Conclusions}

A non-idealized photon-electron detailed balance model has been developed to calculate the realistic intrasubband and intersubband transition currents in a real QD-IBSC prototype in the radiative limit. Instead of assuming absolute photon absorption, as is done in idealized detailed balance models, the model assumes realistic absorption, taking as input the optical matrix elements calculated in previous works.

The model has been used to reproduce experimental measurements of the IQE at different temperatures, with good agreement. This validates the model for calculations at short circuit, where radiative processes dominate, and indicates how the model can be refined for better agreement. It has also allowed a deeper analysis of measured phenomena, uncovering mechanisms that are difficult to uncover experimentally. We believe the model can be useful for evaluating future QD-IBSC proposals, and therefore have provided a full derivation in this article.

The most convincingly reproduced result is the temperature decay of the first IQE peak (interpreted as the $\mathrm{VB} \rightarrow \mathrm{IB}(111)$ 
transition). Two regimes are disclosed: at room temperature, the IQE is limited by the VB $\rightarrow$ IB transitions pumped by the monochromator, and at temperatures below 150-200 K, the IQE is limited by thermal escape from the IB to the CB.

The results confirm quantitatively that the measured thermal escape from the QD confined levels ${ }^{[13]}$ can be entirely accounted for by the absorption of thermal photons, incident from the surroundings and from the substrate, in a sequential many-photon absorption/emission process involving the ladder of QD excited states between the QD ground state and the CB. This mechanism was proposed in a previous paper. ${ }^{[15]}$ This process, when dominant, fundamentally prevents QD-IBSCs from delivering positive sub-bandgap current at high voltages, and prevents QD-IBSCs from having higher efficiencies than equivalent single-gap reference devices, ${ }^{[19]}$ even if the cell is perfectly manufactured. It is therefore concluded that future QD-IBSC prototypes must be designed to eliminate this escape path. One possible option is the use of higher bandgap hosts ${ }^{[20,21]}$ and smaller quantum dots ${ }^{[23]}$ This combination could present a QD ground state deep in the forbidden band with no intermediate excited states between it and the CB.

The results also reveal the sub-bandgap photogeneration is in fact a sequential three-photon process requiring photons with energy as low as $100 \mathrm{meV}$ for the lowest energy transition. This could explain the difficulty of measuring the intrasubband transitions in the past.

Finally, weak photon absorption by intermediate transitions is a serious limitation of the QD-IBSCs. Increasing this absorption should form an important part of QD-IBSC research.

\section{Supporting Information}

Supporting Information is available from the Wiley Online Library or from the author.

\section{Acknowledgements}

This paper is partially sponsored by the European Commission NGCPV (283798) and the Madrid Regional Government grant NUMANCIA-2 (S2109/ENE1477). The authors thank Iñigo Ramiro for useful comments.

Received: May 3, 2013

[1] W. Shockley, H. J. Queisser, J. Appl. Phys. 1961, 32, 510.

[2] a) R. T. Ross, A. J. Nozik, J. Appl. Phys. 1982, 53, 3813; b) J. M. Luther, M. C. Beard, Q. Song, M. Law, R. J. Ellingson, A. J. Nozik, Nano Lett. 2007, 7, 1779

[3] A. Luque, A. Martí, Phys. Rev. Lett. 1997, 78, 5014.
[4] A. Luque, A. Marti, C. Stanley, N. Lopez, L. Cuadra, D. Zhou, J. L. Pearson, A. McKee, J. Appl. Phys. 2004, 96, 903.

[5] a) S. M. Hubbard, C. D. Cress, C. G. Bailey, R. P. Raffaelle, S. G. Bailey, D. M. Wilt, Appl. Phys. Lett. 2008, 92, 123512; b) S. Blokhin, A. Sakharov, A. Nadtochy, A. Pauysov, M. Maximov, N. Ledentsov, A. Kovsh, S. Mikhrin, V. Lantratov, S. Mintairov, N. Kaluzhniy, M. Shvarts, Semiconductors 2009, 43, 514.

[6] a) V. Popescu, G. Bester, M. C. Hanna, A. G. Norman, A. Zunger, Phys. Rev. B 2008, 78, 205321; b) R. Oshima, A. Takata, Y. Okada, Appl. Phys. Lett. 2008, 93, 083111.

[7] A. Luque, P. G. Linares, E. Antolin, E. Canovas, C. D. Farmer, C. R. Stanley, A. Marti, Appl. Phys. Lett. 2010, 96, 013501

[8] a) A. Luque, A. Marti, N. Lopez, E. Antolin, E. Canovas, C. Stanley, C. Farmer, L. J. Caballero, L. Cuadra, J. L. Balenzategui, Appl. Phys. Lett. 2005, 87, 083505; b) A. Martí, E. Antolín, C. R. Stanley, C. D. Farmer, N. López, P. Díaz, E. Cánovas, P. G. Linares, A. Luque, Phys. Rev. Lett. 2006, 97, 247701.

[9] A. Luque, A. Marti, E. Antolin, P. Garcia-Linares, Sol. Energy Mater. Sol. Cells 2010, 94, 2032.

[10] A. Luque, A. Martí, A. Mellor, D. Fuertes Marrón, I. Tobías, E. Antolín, Prog. Photovoltaics 2013, 21, 658.

[11] a) A. Luque, A. Martí, E. Antolín, P. G. Linares, I. Tobías, I. Ramiro, E. Hernandez, Sol. Energy Mater. Sol. Cells 2011, 95, 2095; b) A. Luque, A. Mellor, E. Antolín, P. G. Linares, I. Ramiro, I. Tobías, A. Martí, Sol. Energy Mater. Sol. Cells 2012, 103, 171.

[12] A. Luque, A. Mellor, I. Ramiro, E. Antolín, I. Tobías, A. Martí, Sol. Energy Mater. Sol. Cells 2013, 115, 138.

[13] E. Antolin, A. Marti, C. D. Farmer, P. G. Linares, E. Hernandez, A. M. Sanchez, T. Ben, S. I. Molina, C. R. Stanley, A. Luque, J. Appl. Phys. 2010, 108, 064513.

[14] E. Antolin, A. Marti, P. G. Linares, I. Ramiro, E. Hernandez, C. D. Farmer, C. R. Stanley, A. Luque, in 35th IEEE Photovoltaic Specialists Conference (PVSC), Honolulu, HI, USA 2010, p 000065.

[15] A. Luque, A. Marti, E. Antolin, P. G. Linares, I. Tobias, I. Ramiro, AIP Adv. 2011, 1, 022125

[16] A. Luque, P. G. Linares, E. Antolín, I. Ramiro, C. D. Farmer, E. Hernández, I. Tobías, C. R. Stanley, A. Martí, J. Appl. Phys. 2012, $111,044502$.

[17] A. Luque, A. Mellor, I. Tobías, E. Antolín, P. G. Linares, I. Ramiro, A. Martí, Phys. B 2013, 413, 73.

[18] A. Luque, A. Marti, IEEE Trans. Electron Devices 2010, 57, 1201.

[19] A. Luque, A. Marti, L. Cuadra, IEEE Trans. Electron Devices 2001, 48, 2118.

[20] I. Ramiro, E. Antolin, M. J. Steer, P. G. Linares, E. Hernandez, I. Artacho, E. Lopez, T. Ben, J. M. Ripalda, S. I. Molina, F. Briones, C. R. Stanley, A. Marti, A. Luque, in 38th Photovoltaic Specialists Conference (PVSC), IEEE, Austin 2012

[21] E. Antolín, A. Martí, A. Luque, in 37th Photovoltaic Specialists Conference, IEEE, Seattle 2011.

[22] P. G. Linares, A. Marti, E. Antolin, A. Luque, J. Appl. Phys. 2011, 109 014313.

[23] A. Mellor, A. Luque, I. Tobias, A. Marti, Appl. Phys. Lett. 2012, 101, 133909.

[24] G. Bastard, U. O. Ziemelis, C. Delalande, M. Voos, A. C. Gossard, W. Wiegmann, Solid State Commun. 1984, 49, 671.

[25] P. Wurfel, J. Phys. C 1982, 15, 3967. 